\title{
Feasibility of Cervical Epidural Anesthesia for Breast Cancer Surgery
}

\author{
Manuel Wenk,, ${ }^{1}$ Christina Massoth, ${ }^{1}$ Daniel M. Pöpping,, and Michael Möllmann² \\ ${ }^{1}$ Department of Anesthesiology, Intensive Care and Pain Medicine, University Hospital Muenster, Muenster, Germany \\ ${ }^{2}$ Department of Anesthesiology and Intensive Care, St. Franziskus Hospital, Muenster, Germany \\ Correspondence should be addressed to Manuel Wenk; manuelwenk@uni-muenster.de
}

Received 20 March 2017; Accepted 14 June 2017; Published 18 July 2017

Academic Editor: Enrico Camporesi

Copyright (c) 2017 Manuel Wenk et al. This is an open access article distributed under the Creative Commons Attribution License, which permits unrestricted use, distribution, and reproduction in any medium, provided the original work is properly cited.

\begin{abstract}
Background. Effects of perioperative cervical level neuraxial blocks on the dissemination of cancer metastases have become a matter of substantial interest. However, experience with these catheters has been limited and data on feasibility and efficacy is sparse. Methods. Data from 39 patients scheduled to undergo breast cancer surgery while awake with a cervical epidural alone was retrospectively analyzed. Results. In 26 patients $(66,7 \%, 95 \%$ CI 51,7-81,7) the cervical epidural catheter was sufficient for surgery. In one patient $(2.6 \%, 95 \%$ CI $0-7.6)$ identification of the epidural space was not possible. Four patients $(10.3 \%, 95 \%$ CI $0,7-19,9)$ had an insufficient sensory block. Seven patients (17.9\%, 95\% CI 5,7-30,1) had a partially insufficient sensory block. Rates of failed epidural blocks were not significantly different between different insertion levels. 21 patients $(80.8 \%, 95 \%$ CI 65,4-96,1) developed hypotension and required an intravenous vasopressor. One patient developed nausea. In one patient the dura was accidentally punctured. No neurological damage was observed. No other major complications were observed. Discussion. Epidural punctures in the cervical region are feasible but do bear potential for major complications. Anesthesiologists should familiarize themselves with high epidural block techniques.
\end{abstract}

\section{Introduction}

Cancer remains one of the leading causes of death in developed countries. While lung cancer is predominant in men (17\%), women suffer most frequently from breast cancer (23\%) [1]. While the primary tumor can be removed in almost all cases, the dilemma is that $90 \%$ of all cancer patients die from metastatic disease [2]. Over the last couple of years the role of a perioperatively compromised immune system leading to easier dissemination of tumor cells and accelerated growth of micrometastases has been extensively discussed [3-5]. It appears that reduced perioperative stress might transfer into a better immune response of the patient; hence anesthetic techniques per se have become a significant field of research [6-9]. Particularly the effects of epidural anesthesia have been the center of attention since neuraxial blocks generally provide excellent analgesia and potentially reduce perioperative stress resulting in an overall reduction of the requirement for other anesthetic and analgesic agents in the postoperative period [10-12]. However, despite intensified research, clinical evidence for a potentially protective effect of regional anesthetic and analgesic techniques with respect to cancer recurrence and the incidence of metastases after tumor surgery is still inconclusive [13].

Consequently, with no effect of regional anesthetic techniques known to promote the growth of tumor or metastases, it appears that these techniques should be widely employed whenever possible. However, particularly in the case of breast cancer surgery, a sufficient epidural technique requires the insertion of a cervical epidural catheter at the $\mathrm{C} 6 / \mathrm{C} 7 / \mathrm{T} 1$ level. In stark contrast to the high volume of breast cancer surgery performed in many hospitals stands the relatively rarely performed anesthetic technique of cervical epidural catheter insertion, which both can be challenging for the anesthetist and involves potentially devastating sequelae for the patient. Traditionally, cervical epidural catheters have only been used in selected centers performing cardiac or other high risk surgery; hence the familiarity of anesthetists with this 
technique is not necessarily ubiquitous. The available data on feasibility and efficacy of cervical epidural anesthesia is sparse, consisting mainly of case reports reporting complications and a few studies from the field of pain medicine $[14,15]$.

In this retrospective study we sought to verify the feasibility in terms of success/failure rates and investigated complications in a series of patients undergoing breast cancer surgery while awake with a cervical epidural catheter.

\section{Methods and Materials}

After approval from the Ethics Committee of the University of Muenster and the Medical Board of North-Rhine Westphalia (protocol number $2012415 \mathrm{fN}$ ), data from 39 consecutive women planning to undergo breast cancer surgery while awake with only a cervical epidural catheter and no general anesthesia was retrospectively analyzed.

Briefly, patients with a normal coagulation profile (INR $<1.4$, aPTT $<41 \mathrm{sec}$, and platelets $>80.000$ per microliter of blood) were connected to standardized anesthetic monitoring, including electrocardiogram, noninvasive blood pressure with a 3 min cycle period, and peripheral oxygen saturation. An invasive blood pressure monitoring was used based on the patient's individual risk factors. All patients received a standardized cervical epidural catheter insertion by the same anesthesiologist preoperatively. Patients were placed in the lateral position with their heads anteflexed. The seventh cervical vertebra was marked as an orientation landmark and the area was thoroughly cleaned and disinfected. Operator was using sterile gloves, gown, and face mask for all procedures. The area was covered with sterile draping and the skin and subcutaneous tissue were infiltrated with 2-5 ml prilocaine $1 \%$ (Xylonest 1\%, Astra Zeneca, Germany). The epidural space was punctured at the vertebrae level C6/C7 or C7/T1 or T1/T2 with a standard 18-gauge Tuohy needle (B. Braun, Melsungen, Germany) using a midline approach with a loss of resistance to air technique. An epidural catheter was then inserted via the Tuohy needle and advanced cranially in the epidural space for $2 \mathrm{~cm}$. The Tuohy needle was then removed and the catheter was fixated and covered with sterile dressings. A bolus of $10 \mathrm{ml}$ ropivacaine $0.75 \%$ (Astra Zeneca, Hamburg, Germany) was administered. Patients were then turned supine and after 7-10 minutes a further 3-5 $\mathrm{ml}$ ropivacaine $0.75 \%$ was given. Sufentanil (Actavis, Munich, Germany) was available to be used for epidural application at the anesthetist's discretion. Surgery was then started following a 20- to 30minute wait. Postoperatively, the catheter was removed and after fading of the effects of the epidural anesthesia, patients were discharged from recovery and followed up two hourly for 12 postoperative hours.

Data with regard to patient and surgical characteristics, operator experience, site of insertion, and complications as well as the use of cardiovascular drugs was collected. Complications were divided into none, minor, major, or failed attempt. The following complications were defined as minor ones: inability to reach the epidural space, multiple attempts, change of insertion site or a second operator, bleeding from the epidural needle or blood in the epidural catheter, and
TABle 1: Demographic data.

\begin{tabular}{lc}
\hline Age (yrs) & $71.5 \pm 16.0$ \\
Weight $(\mathrm{kg})$ & $71.8 \pm 12.8$ \\
Height $(\mathrm{cm})$ & $165 \pm 6$ \\
$\begin{array}{l}\text { BMI }\left(\mathrm{kg} / \mathrm{m}^{2}\right) \\
\text { Mastectomy (number of patients) }\end{array}$ & $25.0 \pm 7.5$ \\
$\begin{array}{l}\text { Breast conserving surgery } \\
\text { (number of patients) }\end{array}$ & 24 \\
$\begin{array}{l}\text { Secondary breast reconstruction } \\
\text { (number of patients) }\end{array}$ & 12 \\
$\begin{array}{l}\text { Number of epidural punctures at level } \\
\text { C6/C7 }\end{array}$ & 3 \\
$\begin{array}{l}\text { C7/T1 } \\
\text { T1/T2 }\end{array}$ & 27 \\
$\begin{array}{l}\text { Invasive blood pressure monitoring } \\
\text { (number of patients) }\end{array}$ & 9 \\
\hline
\end{tabular}

difficulties advancing the epidural catheter as well as nausea, vomiting, or vertigo.

Dural puncture, nerve injury or any other neurological complications, dyspnea or difficulty in breathing as well as significant hemodynamic depression (systolic blood pressure $<90 \mathrm{mmHg}$ and/or heart rate $<50$ or $>120$ beats per minute after injection of local anesthetic), or any other significant events were considered major complications.

All data was entered into a customized database. Statistical analysis was performed using SPSS Statistics 18.0 (SPSS Inc., Chicago, IL). Categorical variables are expressed as frequency and percentage, whereas continuous variables are represented as means with standard deviation. The nonparametric patients' baseline characteristics were assessed using the Kruskal-Wallis test. Differences were considered statistically significant at $p<0.05$.

\section{Results}

39 patients aged 72 years (range: 29-92) underwent breast cancer surgery while awake using a cervical epidural catheter as the sole method of anesthesia. Demographic and surgeryrelated data of the patient population is shown in Table 1.

In 26 patients $(66,7 \%, 95 \%$ CI $51,7-81,7)$ the cervical epidural catheter was sufficient as the sole method of anesthesia to perform breast cancer surgery.

In one patient $(2.6 \%, 95 \%$ CI $0-7.6)$ identification of the epidural space was not possible and the procedure was aborted. Four patients $(10.3 \%, 95 \%$ CI 0,7-19,9) had an insufficient sensory block leading to a conversion of anesthetic method from cervical epidural catheter to general anesthesia. Seven patients $(17.9 \%, 95 \%$ CI 5,7-30,1) had a partially insufficient sensory block and anesthesia had to be supplemented with intermittent IV application of S-ketamine and/or midazolam.

Rates of total and partially insufficient epidural blocks were not significantly different between the three catheter insertion levels (C6/7 versus C7/T1 versus T1/T2). 
To investigate the effect of a possible operator learning curve, the first 20 cervical epidural punctures were compared with the following 19 punctures. No significant changes in success/failure rates were observed.

21 of the 26 patients $(80.8 \%, 95 \%$ CI $65,4-96,1)$ with a sufficient cervical epidural received at least one dose of an IV vasopressor after epidural puncture due to hypotension. 16 patients received fractionated doses of $20 \mathrm{mg}$ cafedrine and $1 \mathrm{mg}$ theodrenaline (Akrinor ${ }^{\circledR}$; AWD.pharma, Dresden, Germany), three patients received intermittent doses of 5-10 $\mu \mathrm{g}$ nor-epinephrine (Arterenol ${ }^{\circledR}$, Sanofi-Aventis, Frankfurt, Germany), and two patients received a combination of the two drugs. Reflex bradycardia was observed but no intervention was necessary. No patient developed a significant isolated bradycardia.

One patient received an epidural injection of $5 \mu \mathrm{g}$ sufentanil in addition to the local anesthetic and rapidly developed severe nausea and vomiting. No other patient received any additional opioids. One patient developed late ( $>6 \mathrm{hrs}$ ) postoperative nausea and vomiting.

In one patient the dura was accidentally punctured at the $\mathrm{C} 7 / \mathrm{T} 1$ level and the procedure was aborted. No neurological damage or any other sequelae were observed.

No other major complications were observed; particularly, no nerve or other neurological damage was seen during the observation period. No patient suffered from respiratory complications such as dyspnea or apnea.

\section{Discussion}

Our study has shown that cervical epidural anesthesia is a feasible technique with an acceptable success rate that offers sufficient and profound analgesia for breast cancer surgery while patients are awake.

With increasing evidence that neuraxial blocks such as epidural or paravertebral anesthesia may influence the degree of dissemination of metastatic disease $[3,6,7,12,16,17]$ anesthesiologists should no longer consider these techniques as rare methods performed in highly specialized centers. Even if the scientific evidence regarding the precise role of these techniques on cancer recurrence is still questioned by some experts [18], there is no known effect where these methods would in any way promote the spread of cancer cells. Hence the potential benefits in reducing postoperative pain alone appear to outweigh the risks of the method; hence anesthesiologists today find themselves in a situation where they need to discuss why they are not liberally employing these techniques. One obvious reason, particularly in breast cancer surgery, is the requirement of a high cervical level block which can be technically challenging and usually only few anesthesiologists would have had previous exposure to these techniques since they are usually restricted to selected centers. With breast cancer surgery being performed in many hospitals this gives rise to a significant discrepancy between the demand for cervical epidurals and the ability of the staff to perform these techniques.

In our study we were able to site a cervical epidural correctly in $67 \%$ of all patients. Although a failure rate of $32 \%$ may appear high at first glance it should be noted that this reflects epidural catheters that were sufficient to perform a surgical procedure without any further anesthesia necessary. $28 \%$ of patients had a partial block that needed either conversion to general anesthesia or supplementation with other analgesics/hypnotics. As such, the complete failure rate was rather low and since epidural blocks and general anesthesia are usually performed in combination it can be speculated that many partially working epidurals would have been considered fully functioning. The combined partial and total failure rates of epidural catheters are comparable to published data. Studies as well as a recent review have highlighted failure rates as high as $32 \%$ for thoracic and $27 \%$ for lumbar epidural catheters, which not only were total failures but also involved partial failures [19, 20]. This raises the important but yet unanswered question whether full or partial blocks do have different effects in their potential influence on intraoperative stress levels as well as on the spread of cancer cells during surgery. Differentiating between a fully and a partially functional neuraxial block may also be an important limitation for all prospective clinical trials currently underway.

Neuraxial anesthesia for breast surgery remains the gold standard in terms of providing postoperative analgesia with some studies even suggesting a reduction in the incidence of postoperative persistent pain, though the quantity of available evidence remains severely limited [21]. As an alternative to cervical epidural anesthesia, thoracic paravertebral block (TPVB) appears like a viable option that has shown some beneficial effects on postoperative pain intensity, perioperative opioid consumption, and prevention of chronic postsurgical pain for patients undergoing breast surgery; however, results vary substantially across studies and there is hardly any data available on the use of TPVB as the sole method of anesthesia for performing breast surgery while patients are awake [22].

We did observe some minor and one major complication. One patient developed severe nausea and vomiting after the epidural application of sufentanil. Close proximity to the area postrema of the brainstem may be a possible cause for this [23]. In one patient the epidural space could not be identified and the procedure was aborted. In one patient the dura was accidentally punctured at the C7/T1 level. Although an unfortunate and stressful event for the anesthetist, no neurological sequelae or other complications were observed. With just one millimeter the epidural space in the cervical region is significantly narrower as compared with the thoracic (4-5 mm) or lumbar (5-6 mm) region. However, since most of the available data being case reports there is a paucity of studies systematically investigating the use of cervical epidural injections and catheters at the cervical level. Some data is available from the field of interventional pain medicine where fluoroscopically guided (cervical) epidural injections are used for managing chronic spinal pain [24]. Although a higher rate of local hematoma was observed as compared to thoracic or lumbar epidurals, no major complications were reported. Injuries to the dural sac were not separately reported. A possible approach to increasing safety of cervical injections may be a prepuncture anatomy check using ultrasound or live puncturing under ultrasound guidance. A study by Kim et al. showed that a prepuncture anatomy check using ultrasound 
may provide valuable information about skin-space distance and potentially lead to increased safety [25].

Our study has limitations. Firstly, limitations of the retrospective nature of the data apply. Secondly, although this is currently the biggest study investigating cervical epidural blocks in patients undergoing breast cancer surgery, the number of patients is still too small to reliably assess safety. Larger prospective trials and data acquisition through network databases are necessary. Thirdly, we did not investigate the effects of varying local anesthetics or other supplements.

It is conceivable that once these techniques are employed more liberally we will see a significant increase in major complications. Therefore, a systematic in-hospital approach to detecting complications is highly recommended and agreements between anesthesia, neurosurgery, radiology, and neurology should be in place to provide sufficient emergency services in case of catastrophic sequelae. Furthermore, all data derived from these patients should be added to a national or international regional anesthesia network database to ensure that we as anesthesiologists get a better understanding of what risks these techniques may hold once they are employed more liberally.

\section{Conclusions}

Based on our data and previously published data from the field of pain medicine, epidural punctures in the cervical region are feasible methods with a failure rate comparable to thoracic or lumbar epidural punctures. Based on the available data they appear to be relatively safe since major complications such as nerve or spinal cord damage are rarely reported events. However, these are rarely performed techniques and it remains questionable whether safety concerns will change once these techniques are employed more liberally on a wider basis by heterogeneous operators. Data from prospective trials and regional anesthesia network databases are necessary.

Nevertheless, in the light of the current evidence regarding the effects of regional block on cancer recurrence anesthesiologists should be concerned with high epidural and other neuraxial techniques. It may still be too early to tell but if there is any relevant effect on cancer recurrence these anesthetic techniques will become the gold standard and it may become difficult to argue why these techniques should not be employed.

\section{Conflicts of Interest}

The authors declare that they have no conflicts of interest.

\section{Authors' Contributions}

Manuel Wenk designed the study, interpreted the data, and drafted the manuscript. Christina Massoth contributed to the design of the study and analyzed the data from the databases. Daniel M. Pöpping designed the study, analyzed the data, and participated in drafting the manuscript. Michael Möllmann initiated and coordinated the study and participated in interpretation of the data. All authors read and approved the final manuscript.

\section{References}

[1] A. Jemal, F. Bray, M. M. Center, J. Ferlay, E. Ward, and D. Forman, "Global cancer statistics," CA: A Cancer Journal for Clinicians, vol. 61, no. 2, pp. 69-90, 2011.

[2] G. P. Gupta and J. Massagué, "Cancer metastasis: building a framework," Cell, vol. 127, no. 4, pp. 679-695, 2006.

[3] S. Ben-Eliyahu, "The promotion of tumor metastasis by surgery and stress: immunological basis and implications for psychoneuroimmunology," Brain, Behavior, and Immunity, vol. 17, supplement 1, pp. S27-S36, 2003.

[4] G. L. Snyder and S. Greenberg, "Effect of anaesthetic technique and other perioperative factors on cancer recurrence," British Journal of Anaesthesia, vol. 105, no. 2, pp. 106-115, 2010.

[5] I. J. Fidler, "The pathogenesis of cancer metastasis: the "seed and soil" hypothesis revisited," Nature Reviews Cancer, vol. 3, no. 6, pp. 453-458, 2003.

[6] G. S. De Oliveira Jr., S. Ahmad, J. C. Schink, D. K. Singh, P. C. Fitzgerald, and R. J. McCarthy, "Intraoperative neuraxial anesthesia but not postoperative neuraxial analgesia is associated with increased relapse-free survival in ovarian cancer patients after primary cytoreductive surgery," Regional Anesthesia and Pain Medicine, vol. 36, no. 3, pp. 271-277, 2011.

[7] A. Gottschalk, J. G. Ford, C. C. Regelin et al., "Association between epidural analgesia and cancer recurrence after colorectal cancer surgery," Anesthesiology, vol. 113, no. 1, pp. 27-34, 2010.

[8] A. Gottschalk, "Continuous wound infusion of local anesthetics: importance in postoperative pain therapy," Anaesthesist, vol. 59, no. 12, pp. 1076-1082, 2010.

[9] M. Tylman, R. Sarbinowski, J. P. Bengtson, A. Kvarnström, and A. Bengtsson, "Inflammatory response in patients undergoing colorectal cancer surgery: the effect of two different anesthetic techniques," Minerva Anestesiologica, vol. 77, no. 3, pp. 275-282, 2011.

[10] G. S. Cheng and B. M. Ilfeld, "A review of postoperative analgesia for breast cancer surgery," Pain Management, vol. 6, no. 6, pp. 603-618, 2016.

[11] M. A. Palomero Rodríguez, L. Suarez Gonzalo, F. Villar Alvarez, C. Varela Crespo, I. Moreno Gomez Limon, and A. Criado Jimenez, "Thoracic epidural anesthesia decreases C-reactive protein levels in patients undergoing elective coronary artery bypass graft surgery with cardiopulmonary bypass," Minerva Anestesiologica, vol. 74, no. 11, pp. 619-626, 2008.

[12] J. Greisen, D. V. Nielsen, E. Sloth, and C.-J. Jakobsen, "High thoracic epidural analgesia decreases stress hyperglycemia and insulin need in cardiac surgery patients," Acta Anaesthesiologica Scandinavica, vol. 57, no. 2, pp. 171-177, 2013.

[13] K. Byrne, K. J. Levins, and D. J. Buggy, "Can anesthetic-analgesic technique during primary cancer surgery affect recurrence or metastasis?" Canadian Journal of Anesthesia, vol. 63, no. 2, pp. 184-192, 2016.

[14] B. Stauber, L. Ma, and R. Nazari, "Cardiopulmonary arrest following cervical epidural injection," Pain Physician, vol. 15, no. 2, pp. 147-152, 2012.

[15] D. S. Kloth, A. K. Calodney, R. Derby, F. P. Lagattuta, C. O’Neill, E. Yurth et al., "Improving the safety of transforaminal epidural steroid injections in the treatment of cervical radiculopathy," Pain Physician, vol. 14, no. 3, pp. 285-293, 2011.

[16] A. K. Exadaktylos, D. J. Buggy, D. C. Moriarty, E. Mascha, and D. I. Sessler, "Can anesthetic technique for primary breast cancer surgery affect recurrence or metastasis?" Anesthesiology, vol. 105, no. 4, pp. 660-664, 2006. 
[17] A. Gottschalk, S. Sharma, J. Ford, M. E. Durieux, and M. Tiouririne, "The role of the perioperative period in recurrence after cancer surgery," Anesthesia and Analgesia, vol. 110, no. 6, pp. 1636-1643, 2010.

[18] G. Haller and P. S. Myles, "Regional block and cancer recurrence: too early to tell," Anesthesiology, vol. 107, no. 2, p. 354, 2007.

[19] J. Hermanides, M. W. Hollmann, M. F. Stevens, and P. Lirk, "Failed epidural: causes and management," British Journal of Anaesthesia, vol. 109, no. 2, pp. 144-154, 2012.

[20] L. B. Ready, "Acute pain: lessons learned from 25,000 patients," Regional Anesthesia and Pain Medicine, vol. 24, no. 6, pp. 499505, 1999.

[21] A. S. Terkawi, S. Tsang, D. I. Sessler et al., "Improving analgesic efficacy and safety of thoracic paravertebral block for breast surgery: a mixed-effects meta-analysis," Pain Physician, vol. 18, no. 5, pp. E757-E780, 2015.

[22] A. Schnabel, S. U. Reichl, P. Kranke, E. M. Pogatzki-Zahn, and P. K. Zahn, "Efficacy and safety of paravertebral blocks in breast surgery: a meta-analysis of randomized controlled trials," British Journal of Anaesthesia, vol. 105, no. 6, pp. 842-852, 2010.

[23] P. R. Bromage, E. M. Camporesi, P. A. C. Durant, and C. H. Nielsen, "Rostral spread of epidural morphine," Anesthesiology, vol. 56, no. 6, pp. 431-436, 1982.

[24] L. Manchikanti, Y. Malla, B. W. Wargo, K. A. Cash, V. Pampati, and B. Fellows, "Complications of fluoroscopically directed facet joint nerve blocks: a prospective evaluation of 7,500 episodes with 43,000 nerve blocks," Pain Physician, vol. 15, no. 2, pp. E143-E150, 2012.

[25] S. H. Kim, K. H. Lee, K. B. Yoon, W. Y. Park, and D.-M. Yoon, "Sonographic estimation of needle depth for cervical epidural blocks," Anesthesia and Analgesia, vol. 106, no. 5, pp. 1542-1547, 2008. 


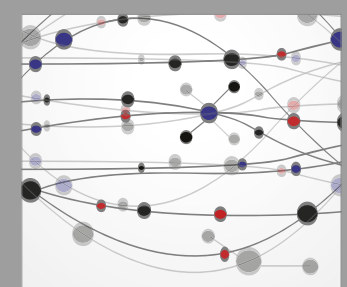

The Scientific World Journal
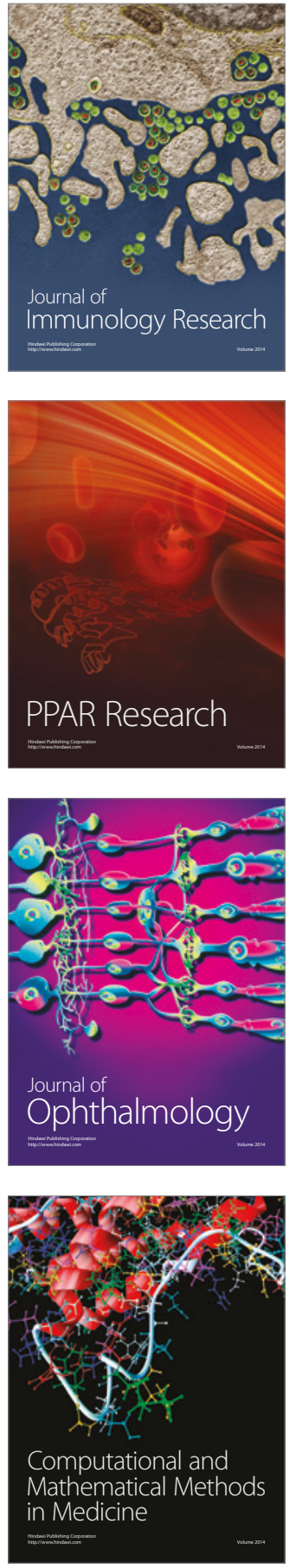

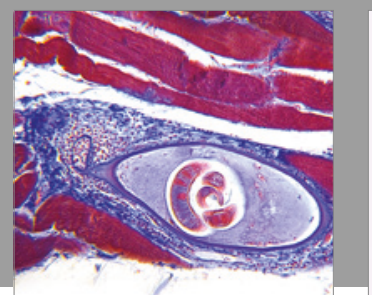

Gastroenterology Research and Practice
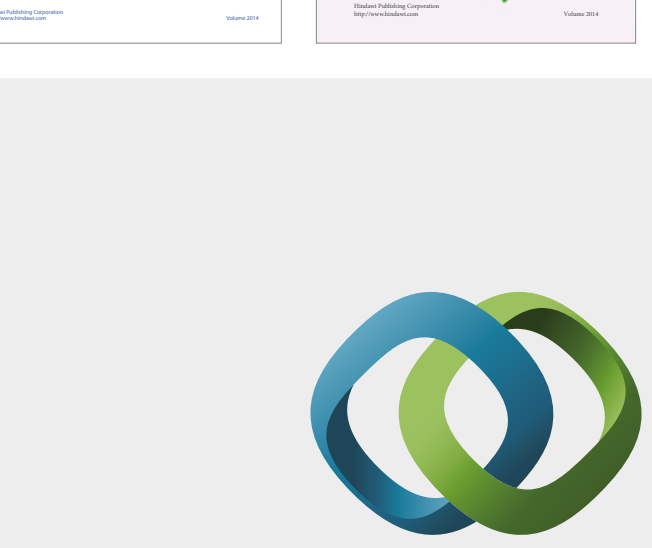

\section{Hindawi}

Submit your manuscripts at

https://www.hindawi.com
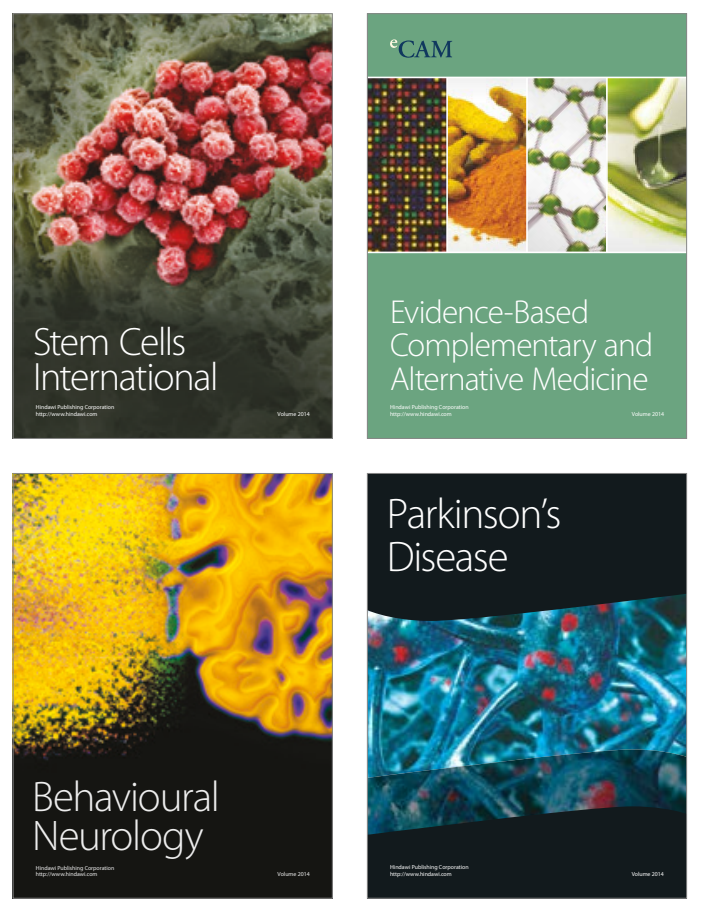
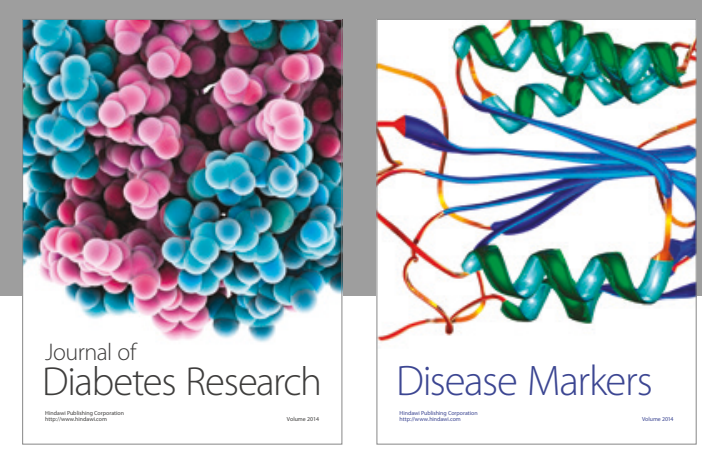

Disease Markers
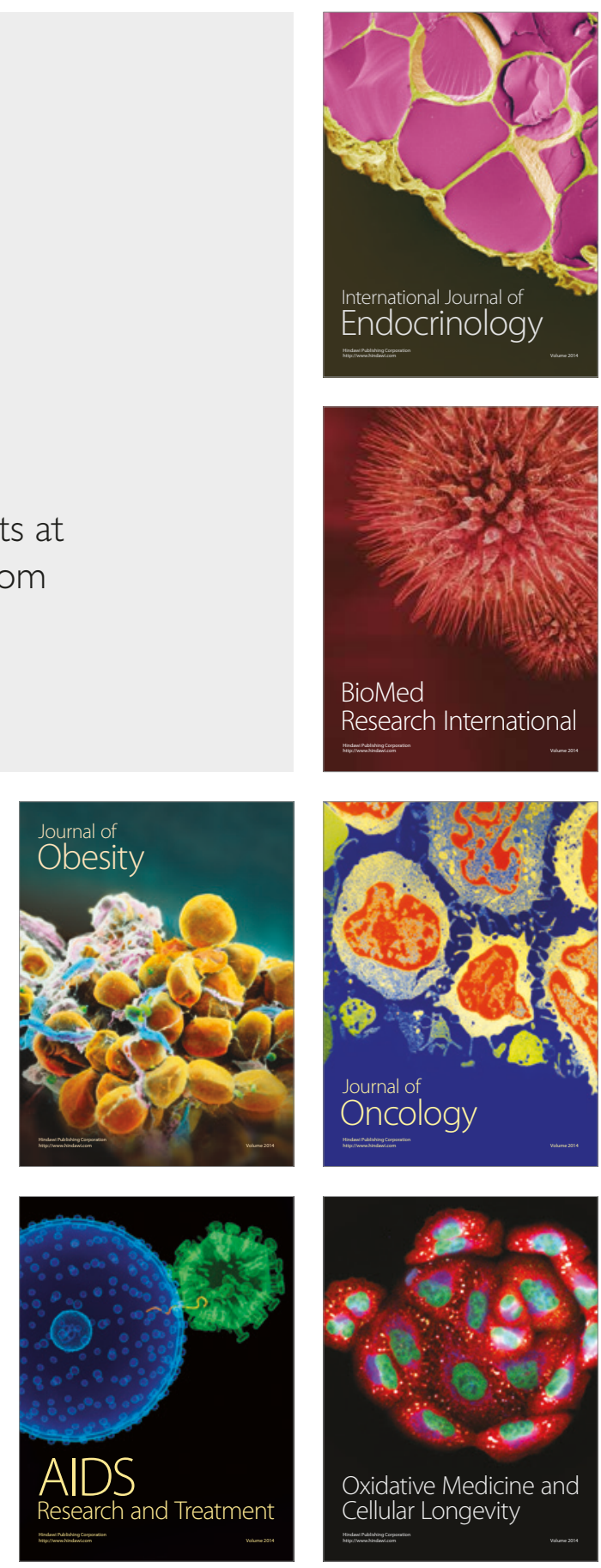